\title{
Estrategias transmedia en las series de televisión: la transformación de una tie-in website en el inicio de un ARG (caso Discover Westworld) Transmedia strategies in TV series: the transformation of a tie-in website in the beginning of an ARG (case Discover Westworld)
}

Sergio Jesús Villén Higueras Universidad de Málaga

\section{Referencia de este artículo}

Villén Higueras, Sergio Jesús (2017). Estrategias transmedia en las series de televisión: la transformación de una tie-in-website en el inicio de un ARG (caso Discover Westworld). En: adComunica. Revista Científica de Estrategias, Tendencias e Innovación en Comunicación, $\mathrm{n}^{0} 14$. Castellón: Asociación para el Desarrollo de la Comunicación adComunica y Universitat Jaume I, 119-140. DOI: http://dx.doi.org/10.6035/2174-0992.2017.14.7

\section{Palabras clave}

Estrategias transmedia; comunicación promocional; series de televisión; páginas webs; tie-in websites; ARG.

\section{Keywords}

Transmedia strategies; promotional communication; TV series; websites; tie in websites; ARG. 


\title{
Resumen
}

La aplicación de estrategias transmedia para promocionar las series de televisión ha transformado el uso de las páginas webs, convirtiéndolas en espacios capaces de construir relatos que expanden los mundos ficcionales. Estas webs, denominadas principalmente como tie-in websites, además, pueden formar parte de una estrategia transmedia más ambiciosa: un juego de realidad alternativa (ARG). Para explorar esta estrategia, se ha efectuado un análisis semiopragmático de Discover Westworld, una tie-in website vinculada a la serie Westworld que, posteriormente, se transforma en el inicio de un ARG promocional. El análisis explora la naturaleza de los espacios comunicativos donde se lleva a cabo esta estrategia, la implicación y la participación de los usuarios en la construcción de significado de las proposiciones comunicativas y los vínculos que dichas proposiciones establecen con las diferentes partes de la serie. Los resultados obtenidos muestran que esta estrategia se desarrolla únicamente en espacios comunicativos digitales con el fin de que todos los usuarios puedan experimentar y participar en la historia. Asimismo, las proposiciones comunicativas de estos espacios digitales permiten a los usuarios tanto interactuar y personalizar algunos contenidos, como participar de diversas formas en la construcción de la historia. Por último, coexisten en estos espacios comunicativos paratextos, que establecen vínculos promocionales directos con la primera temporada; e hipertextos, que promocionan la serie a través de la expansión de su universo ficcional, los cuales, tras finalizar la primera temporada, se pueden considerar también como hipotextos, que sirven como textos-puente para promocionar una futura producción de la serie.

\begin{abstract}
The implementation of transmedia strategies for the promotion of TV series has changed the use of web pages, thus transforming them into spaces capable of creating stories which expand the fictional universes. These websites, mainly known as tie-in websites, may also be part of a more ambitious transmedia strategy: an alternate reality game (ARG). For the purpose of exploring this strategy, it has been undertaken a semiopragmatic analysis of Discover Westworld, a tie-in website linked to the TV series Westworld which, subsequently, has resulted in the beginning of a promotional ARG. The analysis explores the nature of the communicative spaces where this strategy is implemented, the involvement and the participation of users in creating meanings of the communicative proposals and the links established by these proposals with the different parts of this TV series. The results show that this strategy is entirely developed in digital communicative spaces in order to enable users to fully experience and become part of the story. Likewise, the communicative proposals of these digital spaces allow users to both interact and customize some content, and variously participate in constructing the story. Finally, within
\end{abstract}


these communicative spaces there are paratexts, that provide direct promotional links with the first season; and hypertexts, that promote the series by expanding its fictional universe, which, once the first season has finished, in turn, might be considered as hypotexts that serve as textsbridge for promoting a future production of this series.

\section{Autor}

Sergio Jesús Villén Higueras [sergio.vihi@gmail.com] es doctor con la mención europea por la Universidad de Málaga. Miembro del grupo de investigación “Metodologías y herramientas para la investigación sobre cultura visual”. Áreas de investigación: promoción de cine, televisión y videojuegos, transmedia y aplicaciones. 


\section{Introducción}

En la última década, las series han encontrado en las narrativas transmedia (NT) una forma de contar historias que se adapta mejor al nuevo escenario mediático y a las nuevas exigencias del público. Además, la competencia en este sector no para de crecer, por lo que las NT son también una forma de diferenciarse del resto de producciones y de construir estrategias promocionales que, «a la larga, agudizan el compromiso del público» (Jenkins, Ford y Green, 2015: 160). Su aplicación como estrategia promocional supone el traspaso de un modelo basado en la paratextualidad, en el que todos los contenidos promocionales son un reflejo estético y atractivo de una determinada serie, a uno fundamentado en textos complementarios y autosuficientes que funcionan como entradas al mundo narrativo (Jenkins, 2003).

El uso de las NT también impulsa la construcción, gestión y promoción de universos ficcionales que se transforman en valiosas marcas. Series como Lost (2004-2010), Heroes (2006-2010), The Walking Dead (2010-actualidad) o Games of Thrones (2011-actualidad), entre otras, son ejemplos de cómo utilizar las NT para construir universos y hacer de ellos marcas atractivas.

Cabe destacar, no obstante, que muchas series utilizan las NT tras el éxito de una o varias de sus temporadas. Su implantación desde el inicio de una serie es una opción aún muy poco explorada que, a día de hoy, muy pocas producciones de gran relieve se han aventurado a utilizar. La planificación de una serie, por tanto, no parte generalmente de una concepción transmedia, sino que esta se desarrolla en función del éxito cosechado, la envergadura del universo ficcional y los tipos de público establecidos. Es por ello que uno de los grandes retos en este ámbito es la creación de estrategias transmedia nativas, es decir, «diseñar un mundo y una experiencia transmedia desde el origen» (Costa, 2013: 563).

Actualmente, el uso de estrategias transmedia en las series de televisión se puede observar cada vez más a través de las diversas páginas webs que utilizan estas producciones para promocionarse. Aunque las webs suponen un ámbito muy poco explorado por la escasa originalidad de los contenidos que solían ofrecer, en la actualidad, están incorporando progresivamente nuevas proposiciones comunicativas que aprovechan las posibilidades interactivas del medio y profundizan en los mundos ficcionales con el fin de generar nuevas experiencias en los usuarios (Villén Higueras, 2016).

\subsection{Las tie-in websites como estrategia transmedia}

Para hacer de las páginas webs promocionales un espacio de creación, en lugar de espacios superficiales carentes de significado propio como suelen ser las páginas webs oficiales, algunas series han optado por utilizar en sus estrategias transmedia páginas webs alternativas denominadas principalmente como tiein websites, aunque también se les conoce como fake websites, fictional websites 
o in-universe websites. Como sugieren estos nombres, se trata de páginas webs que promocionan una serie desarrollando la historia que esta propone. Su finalidad no solo radica en captar la atención del público, sino que también pretenden generar un cierto realismo en la historia. La web se convierte así en un medio capaz de contar historias en lugar de ser un simple recipiente de paratextos.

El uso de tie-in websites se ha extendido a todo tipo de producciones culturales como los cómics, la música o la literatura, pero son más utilizadas en el ámbito de los videojuegos, el cine o las series de televisión. En estas industrias es común adquirir numerosos dominios en torno a sus producciones con el objetivo de preservar y controlar todas aquellas páginas webs vinculadas a un universo ficcional. Esta estrategia, inicialmente de carácter disuasorio y preventivo para evitar webs perniciosas con dominios vinculados a sus producciones, ha puesto a disposición de las estrategias transmedia un gran número de posibles tie-in websites. Si bien muchos de estos dominios permanecen desactivados o redirigen a los usuarios hacia una web oficial, otros se utilizan para desarrollar y expandir algunas historias vinculadas a una serie.

Existen numerosos ejemplos de tie-in websites que muestran el potencial promocional de esta estrategia transmedia. Por ejemplo, la serie The Big Bang Theory (2007-actualidad) lanzó la web Penny Blossoms; Breaking Bad (2008-2013) creó la web Save Walter White; Park and Recreation (2009-2015) ha creado la web The City of Panwee, páginas enfocadas en los personajes Ron Swanson (Ron Swanson's Grilling Webpage) y Leslie (Leslie Knope for City Council - 2012) o varias webs en las que se desarrollan las disparatadas ideas de Tom Haverford; y, más recientemente, la serie Silicon Valley (2014-actualidad) ha puesto en marcha numerosas tie-in websites como Pied Piper, Hooli, Raviga o Bachmanity Capital, entre otras, que profundizan en los proyectos de los protagonistas. Estos y otros muchos ejemplos demuestran cómo estas webs pueden ser una buena opción para expandir y trasladar a la realidad las historias de las series.

A diferencia de las webs oficiales, las tie-in websites pueden aparecer en cualquier momento y estar sincronizadas con determinados capítulos o temporadas. En este sentido, Rober Hayes, vicepresidente ejecutivo de medios digitales en la NBC Entertainment, declaraba en relación a las diversas tie-in websites que se pusieron en marcha para promocionar la serie Park and Recreation (2009):

[The web] gives us a platform to extend the characters' storyline beyond that, not only as the season goes on, but between seasons. The whole objective is to have a relationship with the fans. I don't think we realized how powerful it could be, or has become (Robert Hayes citado por Feeney, 2013).

Utilizando esta misma estrategia, la serie How I Meet Your Mother (2005-2014) llegó a crear hasta veinticinco tie-in website (además de blogs y perfiles en las redes sociales) que profundizan en todo tipo de aspectos de esta sitcom. Cada vez que un personaje mencionaba una página web en la serie, por muy disparatada que pareciese, los fans podían visitarla como si fuese real. Como señala Carter Bays, uno de los creadores de esta serie: 
The Internet is so user-accessible that whenever you hear ' $w$ - $w$ - $w$ ' on a show, you just know that there's probably something behind it. There's an extra layer of depth to this show knowing that you can go to a different medium and continue the story (Bays citado por Coyle, 2009).

Aunque el uso de este tipo de páginas webs está cada vez más extendido, rara vez permiten que los usuarios puedan participar e interactuar con los contenidos. A pesar del potencial que ofrecen para contar historias de una forma interactiva, se opta frecuentemente por un modelo clásico en el que predominan bloques de texto, imágenes estáticas y vídeos. Es paradójico, por tanto, cómo en una época en la que los usuarios demandan una mayor participación, muchas de estas estrategias transmedia siguen apostando principalmente por contenidos clásicos en espacios potencialmente interactivos.

Tales expansiones de una historia, asimismo, activan la curiosidad de los usuarios que deben estar atentos a cualquier pista que pueda aparecer en una serie. Al estar normalmente desvinculadas de otras tie-in websites o de la página oficial para potenciar su realismo, su acceso a veces implica una previa exploración en wikis, foros $\mathrm{u}$ otros espacios donde los seguidores analizan los episodios y descubren los vínculos con estas páginas webs.

Pero esta desconexión, sin embargo, no implica que estas webs no puedan mantener un vínculo connotado con otras tie-in websites con fines lúdicos. En ellas podrían hallarse códigos, pistas o contenidos vinculados a juegos de realidad alternativa, conocidos también como ARG (sigla derivada de Alternate Reality Games), lanzados para promocionar una serie.

La forma en la que las tie-in websites han pasado de ser una estrategia transmedia sencilla (simple extensión de la historia) a una más compleja como los ARG (webs vinculadas con textos procedentes de otros medios con fines lúdicos) se puede observar en la mítica serie Doctor Who (2005-actualidad). En 2005, los productores descubrieron que de los contenidos promocionales que generaban, los que más les gustaban a los seguidores eran los «games and those fictional sites» (Goss, 2006:66). Tras el descubrimiento, la serie pasó de tener cuatro tie-in websites en 2005 a elaborar decenas de ellas al año siguiente. Los productores, no obstante, tenían en mente «the most ambitious online ficional world ever» (Goss, 2006:66) y produjeron un ARG que combinaba las dos preferencias que habían detectado en los seguidores. Estas webs, por tanto, dejaron de ser micronarraciones que mantenían vínculos concretos con determinados capítulos y se constituyeron como una malla de webs que se retroalimentaban, construyendo un historia más compleja que generaba un espectro más amplio de sinergias promocionales.

\subsection{La tie-in website como rabbit hole: ARG promocionales de las series}

Hace más de una década que los ARG se introdujeron en las estrategias promocionales de las series de televisión para crear nuevas experiencias en los 
usuarios. Aún marcados por lo experimental, los ARG se presentan hoy en día como uno de los formatos más prometedores capaces de difuminar los límites entre la realidad y la ficción. Es por ello que las industrias culturales, creadoras de mundos ficcionales, están viendo los ARG como portales para transportar sus historias a la realidad. Su uso es cada vez más frecuente aunque, «for the most part, alternate reality games today are small-scale probes of the future» (McGonigal, 2011: 126). Se está gestando, pues, una nueva cultura en torno a los ARG y sus posibilidades promocionales se van ampliando conforme surgen nuevos ejemplos.

Una clara definición de lo que son los ARG la ofrece Scolari (2013: 265) cuando los identifica como «una forma de narrativa transmedia que exige una gran cooperación de los participantes y articula actividades dentro y fuera del espacio mediático». En concreto, cabe destacar que los ARG son una de las múltiples plataformas posibles dentro de un universo transmediático que, al mismo tiempo, son en sí mismos una narración transmedia puesto que utilizan todo tipo de textos diseminados por cualquier medio, plataforma o espacio físico (Ryan, 2013: 77).

Desde una perspectiva lúdica, a diferencia de la mayoría de los juegos que se llevan a cabo en un círculo mágico, entendido este como el espacio en el que tiene lugar un juego (un tablero, una determinada superficie o una pantalla), los ARG desdibujan y sobrepasan los límites establecidos tradicionalmente por dicho círculo mágico, puesto que «the game no longer takes place in certain times or certain places [...], bleeding from the domain of the game to the domain of the ordinary" (Montola, Stenros y Waern, 2009: 12). Esto es posible gracias al despliegue tecnológico de las sociedades hodiernas, que permite fácilmente acceder a Internet desde cualquier punto y posibilita que la gente se comunique en cualquier momento a través de los dispositivos móviles.

Respecto a su funcionamiento, los ARGs comienzan con una o varias pistas (trails) que sirven para iniciar el juego. Dispersas por diferentes medios, incluida la realidad como tal, estas pistas pueden aparecer cifradas en paratextos, webs, blogs, vídeos, correos electrónicos, SMS, audios, textos u objetos en el mundo real para captar la atención del mayor público posible. Estos materiales sirven para establecer el primer punto de entrada en el ARG, al cual se le denomina rabbit hole o trailhead en alusión al cuento Las aventuras de Alicia en el país de las maravillas. Como en este relato, los ARG clásicos suelen utilizar también un solo rabbit hole, aunque la búsqueda de públicos diversos y una mayor participación ha fomentado que algunos ARG comiencen a utilizar múltiples trailhead para que la entrada al universo ficcional sea más permeable (Palmer y Petroski, 2016). En el ámbito promocional, estos agujeros suelen ser, en muchos casos, una tie-in website que detenta nuevos contenidos e información encriptada que permiten estructurar y continuar con el juego.

Un ARG, por tanto, es una historia compuesta por una serie de fragmentos que pueden estar disgregados en diversos medios, la cual debe de ser reconstruida 
por los usuarios. No obstante, aunque los participantes son los que articulan los fragmentos para construir la historia, el rumbo de esta lo decide el puppetmaster que, por lo general, es un diseñador del juego que monitoriza a los participantes y hace los ajustes necesarios para que la historia y la experiencia del juego sea atractiva. El puppetmaster orquesta e incentiva también la búsqueda de nuevos fragmentos, proponiendo la narración como un ejercicio de arqueología.

La dispersión de los fragmentos en cualquier lugar de la Web o espacio físico imposibilita, en la mayoría de los casos, que un usuario pueda por sí mismo reunir todas las piezas del juego. Estos juegos, por tanto, están diseñados como una experiencia colectiva en la que los participantes se organizan e interactúan para superar los retos del juego y avanzar en la historia. Es frecuente, pues, la fundación de comunidades de seguidores que funcionan como motor tanto para expandir aquellos universos ficcionales que les gustan, como para instalarlos en su realidad y cotidianeidad.

Si un ARG persigue cruzar la frontera de la ficción para aproximarse a la realidad de los jugadores, se produce entonces una extracción del universo ficcional. Mientras que otras estrategias promocionales potencian la inmersión de los usuarios en los universos de ficción, los ARG, en cambio, trasladan dichos universos «à l'extérieur du monde diégetique (story world), pour l'intégrer dans la vie ordinaire» (Di Crosta y Chantôme, 2016). Las series, en este caso, pueden promocionarse así alternando estrategias que den forma a un modelo en espiral basado en la inmersiónextracción del universo ficcional.

Son numerosos los ejemplos que han demostrado un gran potencial en los ARG promocionales utilizados en las series de televisión. Ejemplo de ello se observa en The Lost Experience de la serie Lost (2004-2010), Heroes 360 Experience en la de Heroes (2006-2010), The Torchwood Mission Game de Torchwood (20062011), The Hunter Pray para la serie Dexter (2006-2013) o los ARG relacionados con las series Kyle XY (2006-2009) o True Blood (2008-2014), entre otros muchos casos. Todos estos ARG, elaborados para la promoción de estas series, tienen un punto en común: el uso frecuente de tie-in websites que, además, actúan a menudo como rabbits holes.

La creación de este tipo de webs es una opción «low-cost, easily updated, free access point» (Kim et al., 2009), que permite alcanzar a un público masivo y global. Por su naturaleza, la web se constituye como una de las formas de rabbit hole más utilizadas, puesto que «ce point d'entrée de l'ARG constitue le plus souvent aussi le lieu de l'articulation/centralisation entre plusieurs médias ou plates-formes» (Di Crosta y Chantôme, 2016). Asimismo, estos espacios posibilitan la entrada de nuevos jugadores en cualquier momento y facilitan la recolección de datos para monitorizar la participación.

Una vez establecidos los puntos de entrada, los ARG promocionales se pueden focalizar en promocionar el estreno de una determinada temporada (transmedia de apelación), suplir los espacios entre una temporada y otra (transmedia de 
transición) o de forma sincronizada o intercalada con los episodios (transmedia de acompañamiento) de una temporada (Cailler y Lacroix, 2014). Hasta ahora, estas estrategias transmedia difícilmente se prolongan tras acabar la temporada de una determinada serie y, si se requiere usar un ARG en temporadas ulteriores, se crea uno nuevo que aborde otras partes del universo ficcional. Este contexto permite aún explorar nuevas formas de ARG promocionales vinculadas a las series televisivas, cuya dilatación en el tiempo podría ocasionar un ARG permanente en el que se desarrollaran estrategias transmedia mucho más elaboradas y flexibles.

\section{La investigación}

\subsection{Delimitaciones y justificación}

Muchas series emplean tie-in websites con diferentes objetivos promocionales a lo largo de su emisión, aunque muy pocas forman parte de una estrategia transmedia mucho más ambiciosa, siendo en este caso un ARG. Asimismo, aunque este tipo de estrategias se utilizan en series de televisión con un cierto grado de madurez, donde los públicos objetivo han sido identificados previamente y las sinergias promocionales son más amplias con los textos que componen el universo ficcional, también se están empezando a utilizar desde el inicio de una serie, en la que el público y el universo ficcional aún se están definiendo. El interés de esta investigación, por tanto, se centra en aquellas tie-in websites que surgen de forma paralela al estreno de una serie nueva y que, al mismo tiempo, sirven posteriormente como base e inicio de un ARG.

En base a estas delimitaciones, se va a analizar Discover Westworld, una tie-in website que se lanzó de forma paralela al estreno de la serie Westworld (2016). Al final de la primera temporada, esta tie-in website evoluciona y sirve como punto de entrada a un ARG que profundiza y expande la estrategia transmedia llevada a cabo por esta serie.

\subsection{Objetivos e hipótesis}

La finalidad principal de este artículo es explorar esta estrategia transmedia desde una perspectiva promocional. Inicialmente, aunque el uso de una tie-in website como puerta de entrada a un ARG supone que el inicio de esta estrategia se constituye en una realidad digital accesible generalmente para todos los usuarios, su posterior desarrollo puede implicar a la propia realidad como un medio más, intercalando así ambas realidades de los usuarios con diferentes finalidades promocionales. Este mestizaje de realidades, si bien ofrece una experiencia más dinámica, también limita la accesibilidad, ya que generalmente impide que un mismo usuario pueda experimentar en primera persona todos aquellos fragmentos que componen la historia propuesta en el ARG. De ahí que 
el primer objetivo sea analizar promocionalmente la realidad de los medios que utiliza un ARG para construir una historia.

Por otro lado, estas historias adoptan la forma de collage a través de múltiples proposiciones comunicativas que establecen diferentes relaciones con los usuarios, requiriendo en muchos casos la implicación de estos en la construcción de dichas historias. Esta implicación es más profunda aún una vez que una tie-in website pasa a formar parte de un ARG, de manera que los usuarios se convierten en jugadores que participan de diversas formas en esta estrategia transmedia. El segundo objetivo, pues, está enfocado en examinar las posibilidades que tienen los usuarios tanto para configurar los significados de las diferentes proposiciones comunicativas de esta estrategia transmedia, como para participar en la construcción de la historia.

Asimismo, si la promoción de una serie puede estar enfocada en potenciar y generar sinergias promocionales con una o varias temporadas, así como ciertos episodios, estas estrategias transmedia, por consiguiente, pueden establecer diferentes relaciones intertextuales. El último objetivo que se propone es explorar las relaciones intertextuales existentes entre esta estrategia transmedia con la serie Westworld (2016).

En base a estos objetivos se establecen las siguientes hipótesis:

- Hipótesis 1: Un ARG promocional que se constituye en una realidad digital mediante tie-in websites, no fragmenta ni limita la participación de los usuarios, permitiéndoles experimentar de forma completa la historia que propone.

- Hipótesis 2: Este tipo de estrategia transmedia utiliza las propiedades comunicativas de las páginas webs para construir una historia con diversas proposiciones que permitan a los usuarios interactuar y personalizar los contenidos y, a su vez, participar de diversas formas en la construcción de la historia de una manera lúdica.

- Hipótesis 3: Un ARG precedido previamente por una tie-in website lanzada de forma paralela al estreno de una serie permite reforzar la noción de universo ficcional y generar de forma estratégica y progresiva sinergias promocionales con la primera temporada, con sus capítulos y, además, con la temporada subsiguiente.

\subsection{Metodología}

A diferencia de los paratextos, que contribuyen a la construcción de un contexto para facilitar la recepción de un producto, una tie-in website, en cambio, es en sí misma un producto que, aunque con fines promocionales, se desarrolla en un contexto mediado donde convergen los significados procedentes de la producción y aquellos que emanan de la experiencia y la participación de los 
usuarios. Más aún, el posible vínculo de estas webs con un ARG implica una construcción de sentido más compleja, puesto que puede derivar en un proceso de construcción social.

En este contexto, para cumplir con los objetivos propuestos, se ha elaborado un método de análisis que adopta una perspectiva semiopragmática, la cual permite «entrelazar el texto con el contexto con la intención de estudiar el funcionamiento» (Fecé, 2004: 254). Asimismo, esta perspectiva semiopragmática permite transformar la noción de contexto por una mucho más dinámica como es la de espacio comunicativo (Odin, 2011). Como sostiene este autor, esta noción nueva se entiende como el espacio donde se producen «un conjunto de condicionantes que empuja a los actantes (E) y (R) a generar sentido bajo el mismo eje de pertinencia» (Odin, 2011: 39). Extrapolado a las necesidades de este estudio, una tie-in website y un ARG se entenderán como un espacio comunicativo, o un conjunto de estos, donde las estrategias promocionales se configuran en base a la naturaleza de la realidad del espacio comunicativo; la implicación de los usuarios en la configuración de significado de los contenidos y la participación de estos en la construcción de la historia; y los vínculos que establecen las proposiciones comunicativas con la serie de televisión.

Inicialmente, puesto que estas estrategias persiguen instalar los universos ficcionales en la vida cotidiana de la gente, se va a diferenciar en el análisis entre espacios comunicativos llevados a cabo en una realidad digital y una física. Mientras que los contenidos desarrollados en espacios comunicativos asentados en una realidad digital pueden sufrir transformaciones para lograr diferentes objetivos promocionales y posibilitar un holgado acceso de público en cualquier momento, los que se desarrollan en la realidad física, en cambio, generan experiencias mucho más personalizadas, aunque son efímeras, con significados estáticos y reducen el acceso de los usuarios. El espacio comunicativo determinará así la participación y el acceso de los usuarios a los contenidos.

En relación a la configuración de significados, en el análisis de estas estrategias transmedia se va a distinguir entre tres proposiciones comunicativas, atendiendo a su nivel de configuración de sus significados por parte de los usuarios: interactivas, que permiten a los usuarios indagar en sus significados a través de una serie de parámetros preestablecidos; personalizables, que posibilitan una configuración subjetiva por parte del usuario; y lúdicas, que adoptan mecánicas de juego para que los usuarios participen y colaboren entre sí para descifrar nuevos contenidos, determinar sus significados y acoplarlos en el collage de fragmentos que componen la historia.

Adicionalmente, en base a las proposiciones comunicativas lúdicas se van a distinguir y analizar tres tipos de participación: analítica, exploratoria y especializada. En el primer caso se trata de proposiciones bloqueadas por un determinado obstáculo (generalmente suele ser un código), lo cual requiere que los usuarios analicen detalladamente todo tipo de contenidos, incluida la serie, para 
superar dicho obstáculo. En segundo lugar, es frecuente en los ARG que existan contenidos ocultos en ciertos espacios comunicativos como las tie-in websites y, para dar con ellos, es necesario explorar en profundidad estas páginas. Por último, estas estrategias utilizan a menudo ciertos mensajes cifrados que requieren de conocimientos específicos para descodificarlos, propiciando así la participación de usuarios especializados en diversas áreas de conocimiento. Todas estas formas de participación están diseñadas para estimular a un amplio espectro de usuarios y conformar comunidades que colaboren con el fin de darle forma y significado a estas estrategias transmedia.

Por otro lado, estas producciones transmedia pueden establecer múltiples vínculos con aquellos textos que componen una serie (episodios, temporadas o fragmentos concretos) y comparten el mismo universo ficcional, siendo estos esenciales para que exista un flujo continuo de sinergias promocionales. Se explorará, por consiguiente, el contexto de esta estrategia analizando si los contenidos de estas producciones funcionan como paratextos, hipertextos o hipotextos.

En el primer caso, aunque las tie-in websites se consideran como creaciones conectadas a las estrategias transmedia, ello no implica que estas puedan incluir algún paratexto como parte de la nueva historia, quedando vinculado de forma coherente con el resto de contenidos. Se analizará, por tanto, si estos paratextos están vinculados a un capítulo determinado y a una o varias temporadas.

En el segundo caso, si la hipertextualidad es entendida según Genette (1982) como el vínculo que establece un nuevo texto (hipertexto) con un texto anterior (hipotexto), se analizará, pues, las relaciones establecidas entre todos aquellos contenidos de una tie-in website o ARG que expanden el universo ficcional (hipertextos) con aquellas partes de una serie previamente emitidas (hipotextos).

En el tercer caso, de forma inversa, dichos contenidos pueden hacer alusión directa a una futura extensión de la serie, promocionando textos que aún no han sido emitidos o creados, pero que lo serán en un futuro. Al constituirse estos contenidos como hipotextos, actúan de bisagras con extensiones subsiguientes de la serie, siendo a corto plazo, si es con un determinado capítulo, o a mediolargo plazo, si es con una nueva temporada.

\section{Análisis y resultados}

\subsection{Discover Westworld como tie-in website}

De forma paralela a su estreno, la serie Westworld (2016) lanzó www.discoverwestworld.com, una tie-in website que con el transcurrir de la serie se convertiría en el inicio de un ARG. El objetivo de esta primera tie-in website es darle forma al parque ficticio Westworld, simulando la web de un parque de atracciones que alienta a los usuarios a visitar el parque. Los contenidos propuestos se dividen en cuatro apartados que persiguen estrategias promocionales diferentes. 
En el primer apartado se hace una presentación de Westworld con un vídeo de fondo de pantalla en el que se van sucediendo diversas escenas de la serie, invitando así al usuario a prepararse para una futura estancia en el parque. Por consiguiente, el vídeo se puede considerar como un paratexto que introduce a los usuarios en el mundo ficcional y los redirige hacia la serie. Igualmente en el inicio de esta página se invita al usuario a inscribirse con sus datos y correo electrónico para recibir información que le ayude a preparar su estancia en el parque. Al inscribirse, los usuarios reciben periódicamente una newsletter con información y contenidos relacionados con Westworld. Se persigue, pues, un trato directo y personalizado con los usuarios en esta experiencia transmedia.

En el segundo apartado la web destaca las características del parque temático. Es un apartado breve en el que se detallan las experiencias que pueden tener los usuarios en Westworld. Utilizando algunos fotogramas (paratextos) como fondo de pantalla, se exponen a modo de palabras clave con una breve descripción las principales características del parque temático: libertad, felicidad, emoción y evasión. Son experiencias y emociones que describen el universo ficcional de Westworld, pero que están dirigidas al mismo tiempo a los usuarios con el fin de crear una experiencia inmersiva. Esta dualidad queda denotada cuando la web expone en relación a la evasión que ofrece este parque a los usuarios: «Our trully-inmersive experience is always evolving to meet your needs and create a rich world where you rule».

Si los apartados anteriores de esta web son proposiciones comunicativas pasivas que presentan al parque, el tercer apartado, en cambio, permite al usuario interactuar con los contenidos e indagar en el mundo ficcional de Westworld. Concretamente, los usuarios pueden explorar libremente un mapa en el que aparecen todas las localizaciones de Westworld. Dichas localizaciones actúan como hotspots que dan acceso a nuevas capas de información en las que aparecen imágenes e información sobre la localización seleccionada. A través de este aparatado no solo se le da forma al universo ficcional de la serie y orienta a los espectadores en las diversas historias que cohabitan en su interior, sino que también identifica localizaciones que no aparecen en la primera temporada. El mapa también deja abiertas futuras líneas de expansión, constituyéndose como una juntura que articula la primera temporada de la serie con posibles extensiones de este mundo ficcional. 
Imagen 1. Mapa interactivo de Westworld

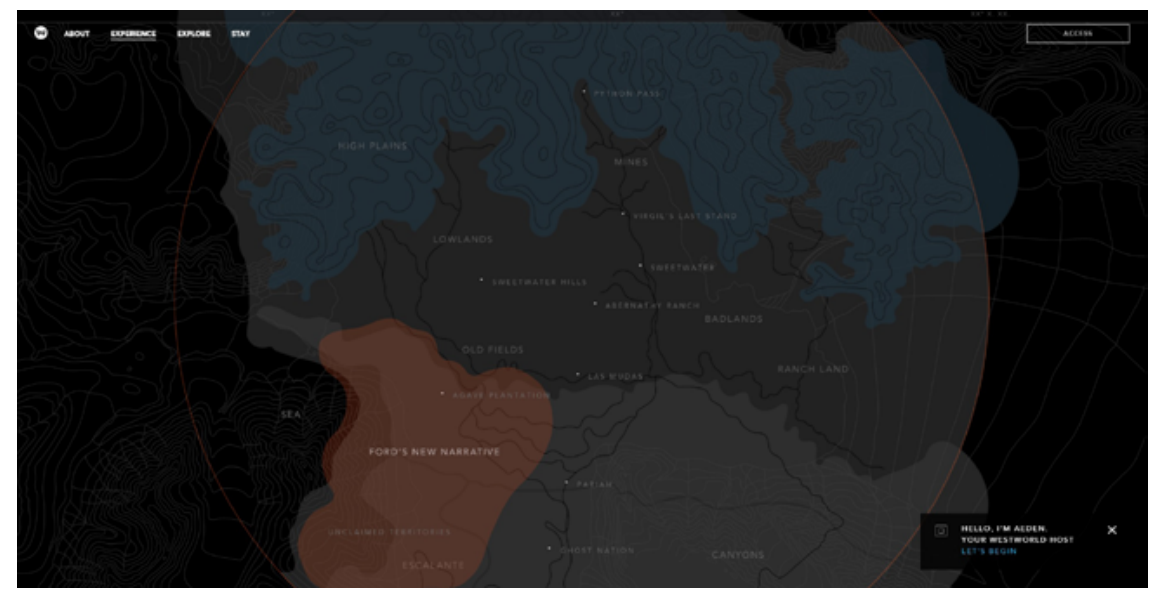

Fuente: página web Discover Westworld

El cuarto apartado de esta web invita directamente a los usuarios a reservar su estancia en Westworld. Para ello se explica de forma detallada el proceso de entrada y salida a Westworld, que forma parte también de la experiencia que ofrece el parque. Tras esta explicación, como si de una compañía de viajes se tratara, esta proposición personalizable ofrece la posibilidad de seleccionar el periodo de la estancia, aunque al efectuar la reserva no hay disponible ninguna fecha y aparece el mensaje "whitehatblackhat», el cual servirá posteriormente como un código que da acceso a nuevos contenidos.

Por último, la web propone adicionalmente otra forma interactiva a través de un chatbot personificado en Aeden, un anfitrión virtual. El chatbot está presente en todo momento en la web para que los usuarios puedan realizar todo tipo de preguntas relacionadas con la serie Westworld en cualquier momento. Su capacidad para responder a las preguntas de los usuarios iba incrementando de forma paralela a la emisión de la serie y sus respuestas, asimismo, no solo están vinculadas a la historia que se desarrolla en la serie de televisión, sino que profundiza también en diversos aspectos del mundo ficcional.

\subsection{Discover Westworld: el inicio de un ARG}

Al finalizar la primera temporada, Discover Westworld deja de ser una simple tie-in website y se convierte en parte de un ARG. Como consecuencia de la situación final del parque, la web es hackeada por un anfitrión, dando acceso a los usuarios a nuevos contenidos que profundizan en la historia y a pistas sobre 
cómo será la segunda temporada. No obstante, los contenidos están ocultos o cifrados por códigos que los usuarios deben encontrar a modo de juego. El ARG favorece así la implicación de los usuarios, en la que es necesaria su participación y su colaboración (por ejemplo, la comunidad creada al respecto en Reddit) para superar los retos que propone este juego. En general, el ARG se divide en dos secciones: una que profundiza en la web Discover Westworld y otra que da acceso a dos tie-in websites vinculadas a la empresa ficticia Delos Incorporated.

Inicialmente, en la parte superior derecha de la web Discover Westorworld hay un punto de acceso que requiere de un código. Tras la exploración de la página por parte de los usuarios, se descubrió que la frase «whitehatblackhat» servía de código y daba acceso a información nueva. Así pues, aparece un vídeo corporativo de Delos Incorporated en el que se presenta Westworld a través de diferentes escenas de la serie, por lo que se puede entender como un paratexto que presenta de forma general al parque y, al mismo tiempo, sirve también como una especie de resumen de la primera temporada. Una vez finalizado el video, se propone un test con veinte preguntas para evaluar a los usuarios y otorgarles un perfil (cazador de recompensas, forajido, sheriff, soldado, etc.) en base a su personalidad. De esta forma se pretende ofrecer una experiencia personalizada para cada individuo y potenciar su vinculación con personajes e historias próximas a su personalidad.

Acto seguido, los usuarios pueden continuar con la reserva de su estancia y seleccionar el número y el tipo de habitación y el tipo de paquete (standard, silver y gold) que determinará las experiencias en el parque. Una vez configurada la estancia, para incrementar el realismo, el usuario recibe una factura en la que se detalla el precio de todos los servicios seleccionados y deberá pagar a través de métodos de pago ficticios. La reserva finaliza cuando el usuario acepta el contrato y los términos de Delos, en el que puede encontrar información complementaria sobre esta empresa y el parque Westworld (participación exploratoria). Los usuarios adoptan así el papel de futuros visitantes que experimentan en primera persona una parte previa de la historia que está omitida en la serie.

De forma complementaria a esta reserva, al finalizar la serie, los usuarios obtuvieron el acceso a una especie de guía de viaje interactiva para una estancia de diez días, coincidiendo con el número de episodios que tiene la primera temporada. El plan para cada uno de los días, además de establecer vínculos con cada uno de los episodios, viene reflejado en fichas en las que se explica y se describe tanto las posibilidades que ofrece el parque como el funcionamiento de la compañía Delos. Además, cada una de las diez fichas contiene varios hipervínculos que redirigen al usuario a fotogramas de la serie, gráficos relacionados con Delos, vídeos con pequeñas escenas de los episodios, un mapa interactivo del parque, el perfil de la serie en Facebook, una lista con las canciones de la banda sonora en iTunes e, incluso, el acceso a algunas partes del sistema informático ficticio de Delos. Esta guía de viaje, por tanto, es una proposición interactiva que entrelaza nuevos contenidos que expanden el universo ficcional con paratextos específicos vinculados a cada uno de los capítulos que componen la primera temporada de esta serie. 
El hackeo a la página web da acceso también a nuevos contenidos, aunque están ocultos para estimular en los usuarios una participación exploratoria. Al pulsar la tecla shift o hacer clic de forma prolongada en la sigla de Westworld en la página de inicio, aparece una especie de vídeo con un bucle de fallos que contiene determinada información sobre la situación final del parque tras finalizar la serie. La transformación de la web en parte de un ARG afecta igualmente al chatbot Aeden, que tiene más capacidad para responder. Aeden evoluciona en esta fase y no solo oculta nueva información que los usuarios deberán sustraer a través de todo tipo de preguntas, sino que sus respuestas van acompañadas de varios hilos de conversación para seguir profundizando en un determinado asunto. A través de Aeden, pues, se propone una experiencia interactiva que permite a los usuarios seguir profundizando en la historia al estilo Elige tu propia aventura.

De forma paralela, esta estrategia potencia también una participación analítica utilizando como contraseña una frase de la serie. El código "violentdelight», que aparece numerosas veces a lo largo de la serie, permite a los usuarios acceder a una nueva tie-in website denominada Delos Destinations (http://www.delosdestinations.com). Al cargar esta nueva página, no obstante, a causa del hackeo producido, sufre una serie de fallos e introduce a los participantes en una tercera tie-in website denominada Delos Incorporated (http://www.delosincorporated.com). Tras una serie de errores ocasionados al entrar en alguna de las secciones de esta web, se accede a una lista de procesos y códigos informáticos que contienen un mensaje cifrado. En este punto, el ARG está orientado a participantes que sustenten conocimientos avanzados en informática para descifrar el mensaje oculto, lo cual incentiva una participación especializada. Al descifrar el mensaje, se desbloquea un clip de audio y otro de vídeo que dan pistas sobre la situación final de un personaje de la serie.

Adicionalmente, en este mismo espacio hay un área de acceso para los administradores que requiere otra vez de una contraseña. Este obstáculo estimula de nuevo una participación analítica en los usuarios, ya que la contraseña aparece cifrada en uno de los correos que recibían todos aquellos usuarios apuntados a esta experiencia transmedia. Una vez hallada la contraseña, se accede al panel de seguridad de Delos, el cual se puede considerar como una especie de intranet de esta empresa ficticia. 
Imagen 2. Panel de seguridad de Delos

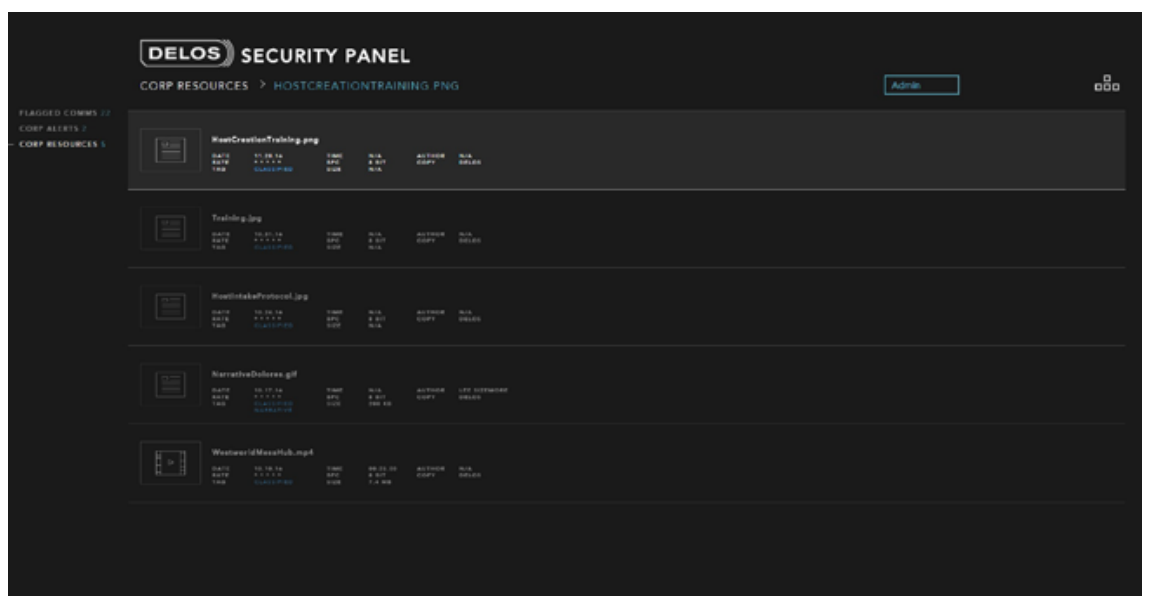

Fuente: página web Delos Incorporated

A través de este panel, los usuarios pueden acceder a correos internos de los trabajadores y archivos multimedia, ambos vinculados a la empresa Delos Incorporated. Estos contenidos, aunque no son proposiciones interactivas, profundizan e indagan en el final de la serie, proporcionando pistas y generando expectativas sobre la siguiente temporada. De forma adicional, integrada en el código fuente de la página, hay también información importante que da pistas a los usuarios sobre el futuro desarrollo de la serie. De esta forma, mientras que el panel de seguridad propone a los usuarios una participación exploratoria, la información oculta en el código fuente estimula una participación especializada.

En resumidas cuentas, este ARG persigue convertir inicialmente a los usuarios en futuros visitantes de Westworld, aunque a causa del ataque que sufre el parque, se alienta a que dichos usuarios aprovechen los fallos en seguridad para conseguir información complementaria a la serie. Para ello se configura una historia fragmentada que contiene ciertas partes codificadas para convertir la narración en un juego. Para convertirse en una estrategia atractiva, este ARG sustenta proposiciones comunicativas interactivas, personalizables y lúdicas que no solo permiten a los usuarios profundizar en la historia y participar de diversas maneras en su construcción, sino que también se convierten en protagonistas de esta experiencia transmedia.

Por otro lado, al desarrollarse en espacios comunicativos digitales, los usuarios pueden experimentar en primera persona y de forma íntegra el ARG. Esta narración transmedia permite concentrar así todas las visitas de los usuarios en estos espacios comunicativos digitales, facilitando el control y la monitorización 
de las visitas, lo cual permite a los creadores del ARG ajustar continuamente la estrategia promocional en base a las acciones de los usuarios. Tales webs, por consiguiente, se configuran como espacios comunicativos idóneos para que los creadores y los usuarios generen sentido bajo el mismo eje de pertinencia.

La naturaleza de estos espacios comunicativos digitales, además, ha permitido ir acumulando e incluyendo progresivamente nuevos contenidos. Se rehúye así de las experiencias efímeras y, en la medida de lo posible, persigue acumular los contenidos utilizados en un mismo espacio para prolongar su existencia incluso una vez finalizado el ARG. De esta forma se favorece que los usuarios puedan experimentar en cualquier momento esta estrategia promocional.

Cabe destacar, no obstante, que todas estas tie-in websites están pensadas como una estrategia nacional, ya que sólo se pueden visitar en Estados Unidos. Así, paradójicamente, espacios comunicativos que potencian la accesibilidad de todos los usuarios para que puedan participar en la experiencia propuesta, sirve a su vez para restringir el acceso a esta experiencia transmedia a un solo territorio.

\subsection{Paratextos, hipertextos e hipotextos}

Al finalizar la primera temporada, Discover Westworld sufre un reciclaje y se convierte en parte de un ARG que, además de seguir profundizando en el universo ficcional, da pistas sobre el desarrollo futuro de la serie. Así pues, en estas estrategias se utilizan contenidos que actúan promocionalmente como: paratextos enfocados en la temporada (video corporativo que actúan como una especie de season trailer) y paratextos enfocados en los capítulos (guía de viaje para diez días que alude a cada uno de los episodios); hipertextos en relación a la primera temporada (chatbot Aeden, mapa interactivo, los contenidos e información de Delos Incorporated, etc.), ya que profundizan y expanden la historia; e hipotextos en relación a la segunda temporada, puesto que todos los contenidos de estas estrategias se pueden considerar también como textos-puente entre la primera temporada y la segunda.

La configuración de todos los contenidos analizados como hipertextos, primero, y como hipotextos, después, muestran la capacidad que tienen estas estrategias transmedia para reciclarse y generar sinergias promocionales multidireccionales. El ejemplo más evidente de esta versatilidad se observa en el chatbot Aeden, el cual ha ido incrementando su capacidad de respuesta de forma progresiva durante y después de la emisión de la serie. De esta forma, Aeden se puede considerar como un hipertexto, ya que profundizaba semana tras semana en el mundo ficcional que se iba generando a través de los capítulos de la serie y, tras la finalización de la temporada, se puede considerar como un hipotexto, puesto que sus respuestas sirven para comprender mejor el final de la primera temporada y, a su vez, para generar expectativas de la próxima. 
$\mathrm{Al}$ acompañar a la serie desde su estreno, esta estrategia origina así un engranaje de textos que se ponen en funcionamiento progresivamente para promocionar la primera temporada, en general, y sus episodios, en particular; para darle forma al universo ficcional a través de su consolidación y expansión; y, por último, para canalizar todas estas sinergias promocionales hacia una futura segunda temporada.

\section{Conclusiones}

El uso de una tie-in website que se convierte de forma estratégica en el inicio de un ARG refleja la importancia de los universos ficcionales como artefacto comunicativo y la capacidad de transformación que tienen estas creaciones para estimular progresivamente las necesidades promocionales de una serie. Las páginas webs, por consiguiente, dejan de ser solamente espacios expositivos henchidos de paratextos y se emplean también como espacios para construir historias que encajan dentro de las estrategias promocionales transmedia de las series de televisión.

Una tie-in website como Discover Westworld, además, se instala en una realidad digital y simula la forma de una web verdadera. La ficción queda así entretejida con la realidad con el fin de sorprender a los usuarios. No obstante, al transformarse en un rabbit hole para iniciar un ARG, la historia se puede complementar con fragmentos desarrollados en la realidad física, construyendo una historia multidimensional. A pesar de esta posibilidad, el ARG analizado apuesta únicamente por una realidad digital, complementando Discover Westworld con otras tie-in websites. Así pues, estos espacios pueden ir acumulando todos los contenidos que se van utilizando a lo largo de la estrategia, lo cual favorece que nuevos usuarios puedan experimentar igualmente la historia propuesta, incluso una vez finalizada esta estrategia.

La construcción de un ARG promocional a través de múltiples tie-in websites, por tanto, no fragmenta ni limita la participación de los usuarios. Sin embargo, pueden estar restringidas a un territorio específico como es el caso de las tie-in websites analizadas. Pese a su potencial para abordar prácticamente a un público global, se ponen de manifiesto las limitaciones de este tipo de estrategias transmedia a nivel internacional.

Por otro lado, la expansión del universo Westworld a través de este tipo de páginas webs se puede llevar a cabo mediante proposiciones comunicativas en las que el usuario sea influyente en la construcción de significados. Así pues, en estas webs se pueden utilizar proposiciones interactivas como el mapa con las localizaciones y el chatbot; proposiciones personalizables como la reserva de una futura estancia en el parque; y proposiciones lúdicas como es el caso de todas aquellas claves, contraseñas y contenidos ocultos que, a lo largo del ARG, propician una participación analítica, exploratoria y especializada. Puesto 
que un mismo usuario difícilmente intervendrá en todas las proposiciones de un ARG, tal diversidad de formas participativas está orientada a la creación de comunidades de usuarios que colaboren para recomponer los fragmentos de esta historia transmedia.

El usuario, asimismo, a lo largo de esta estrategia no sólo es el encargado de reconstruir una historia fragmentada que aplica mecánicas de juego, sino que también influye en la construcción de significado de algunos fragmentos. En esta estrategia convergen así dos grandes retos contemporáneos de la comunicación promocional: la narración transmedia y la implicación y participación de los usuarios en los universos ficcionales.

Estas opciones demuestran la versatilidad y la capacidad que tienen estas webs para construir experiencias inmersivas, que permiten a los usuarios explorar e interactuar con el universo ficcional e, igualmente, también generan experiencias basadas en la extracción, es decir, trasladar la diégesis a un entorno real. Esta extracción, potenciada por la transformación de Discover Westworld en un ARG, persigue transformar a los propios participantes en miembros de un mundo ficticio.

En último lugar, esta estrategia lanzada de forma paralela al estreno de la serie utiliza un conjunto armonizado de hipertextos que refuerzan la noción de universo ficcional y generan de forma intrínseca sinergias promocionales con la serie; incorpora e integra de forma coherente determinados paratextos para establecer vínculos promocionales directos con la serie; y, tras finalizar la primera temporada, se usan hipotextos que actúan como bisagras para promocionar y generar interés en torno a la segunda temporada.

Cabe destacar respecto a los hipotextos que, en general, no son contenidos nuevos, sino hipertextos que, de forma connotada o denotada, transforman y orientan sus significados hacia una nueva temporada. Por ejemplo, el chatbot utilizado en Discover Westworld está orientado inicialmente en responder preguntas para profundizar en asuntos vinculados a la primera temporada pero, tras finalizar la temporada, sufre una transformación y sus respuestas están enfocadas en indagar en la situación final del parque y dar pistas sobre la segunda temporada. Este tipo de webs, por consiguiente, se pueden considerar como un conjunto de textos metamórficos susceptibles de evolucionar y variar sus significados en relación a las necesidades promocionales de una serie.

\section{Referencias}

Cailler, Bruno; Lacroix, Céline Masoni (2014). Transmedia et ARG : Enjeux stratégiques, économie narrative et jouabilité. Le cas In Memoriam. En: Cailler, Bruno; Denis, Sébastien y Sapiega, Jacques (eds.). Histoire du transmédia : Genèse du récit audiovisuel éclaté. Paris: L'Harmattan. 
Costa, Carmen (2013). Narrativas Transmedia Nativas: Ventajas, elementos de la planificación de un proyecto audiovisual transmedia y estudio de caso. En: Historia y Comunicación Social, 18 ( ${ }^{\circ}$ extra 3), 561-574. DOI: 10.5209/ rev_HICS.2013.v18.44349

Coyle, Jake (2009). On the Net: Mock Web sites extend sitcom gags. En: The Sydney Morning Herald. Disponible en: http://www.smh.com.au/technology/ on-the-net-mock-web-sites-extend-sitcom-gags-20090312-8wom.html Consultado $4 / 02 / 2017)$.

Di Crosta, Marida; Chantôme, Ana (2016). La conception de jeux en réalité alternée reliés aux séries télévisées. La scénarisation de fictions ludiques hybrides, entre jeu traditionnel et jeu numérique. En: Sciences du jeu, vol. 5. DOI: $10.4000 /$ sdj. 587

Fecé, Josep Lluis (2004). El circuito de la cultura popular: Comunicación y cultura popular. En: Ardèvol, Elisendra y Muntañola, Nora (coords.). Representación y cultura audiovisual en la sociedad contemporánea (pp. 235-84). Barcelona: Editorial UOC.

Feeney, Nolan (2013). How Parks and Rec Brings Pawnee to Life Online. En: Times. Disponible en: http://entertainment.time.com/2013/02/28/how-parksand-rec-brings-pawnee-to-life-online/ (Consultado 6/02/2017).

Genette, Gérard (1982). Palimpsestes: La littérature au second degré. Paris: Edición du Seuils.

Goss, James (2006). Production Notes: All the, ER, Goss from bbc.co.uk's James Goss. En: Doctor Who Magazine, no 367 (29 de marzo).

Jenkins, Henry (2003). Transmedia storytelling. Moving characters from books to films to video games can make them stronger and more compelling. En: Technology Review. Disponible en: https://www.technologyreview.com/s/401760/ transmedia-storytelling/ (Consultado 20/01/2017).

Jenkins, Henry; Ford, Sam; Green, Joshua. (2015). Cultura transmedia: La creación de contenido y valor en una cultura en red. Barcelona: Gedisa.

Kim, Jeffrey; Lee, Elan; Thomas, Timothy; Dombrowski, Caroline (2009). Storytelling in new media: The case of alternate reality games, 2001-2009. En: First Monday, 14(6). DOI: 10.5210/fm.v14i6.2484

McGonigal, Jane (2011). Reality is Broken: Why Games Make Us Better and How They Can Change the World. New York: The Penguin Press.

Montola, Markus; Stenros, Jaakko; Waern, Annika (2009). Pervasive games: Theory and design. Burlington, Massachusetts: Morgan Kaufmann Publishers.

Odin, Roger (2011). Les espaces de communication: Introduction à la sémiopragmatique. Grenoble: PUG. 
Palmer, Charles; Petroski, Andy (2016). Alternate Reality Games: Gamification for Performance. Londres: CRC Press.

Ryan, Marie-Laure (2013). Transmedial Storytelling and Transfictionality. En: Poetics Today, 34(3), 361-388. DOI: 10.1215/03335372-2325250

Scolari, Carlos Alberto (2013). Narrativas transmedia: Cuando todos los medios cuentan. Barcelona: Deusto.

Villén Higueras, Sergio Jesús (2016). La comunicación promocional en las páginas webs oficiales experimentales del cine comercial hollywoodiense de aventuras: una perspectiva semiopragmática. (Tesis inédita de doctorado). Universidad de Málaga: Málaga. 